Original article

\title{
Exposure risk and COVID-19 infection among frontline health-care workers: A single tertiary care centre experience
}

\author{
Saritha Susan Vargese ${ }^{\mathrm{a}}$, Sahya S. Dev ${ }^{\mathrm{a}}$, Sonu Soman A ${ }^{\mathrm{a}}$, Nisha Kurian ${ }^{\mathrm{a}}$, Abhraham Varghese V ${ }^{\mathrm{b}}$, \\ Elsheba Mathew ${ }^{\text {a, }}$ \\ ${ }^{a}$ Department of Community Medicine, Pushpagiri Institute of Medical Sciences and Research Centre, Tiruvalla, Kerala, India \\ ${ }^{\mathrm{b}}$ Department of General Medicine, Pushpagiri Institute of Medical Sciences and Research Centre, Tiruvalla, Kerala, India
}

\section{A R T I C L E I N F O}

\section{Keywords:}

COVID-19

Frontline health-care workers

Exposure

Risk category

Contact tracing

\begin{abstract}
A B S T R A C T
Background: There is limited data on frontline health-care workers and risk of COVID-19 from the developing nations. It is imperative to identify those at higher risk to prevent further transmission. We assessed the relationship between exposure risk and COVID-19 among front-line health-care workers who were primary contacts of a COVID-19 patient.

Methods: A retrospective cohort study was conducted among front-line health-care workers in a tertiary care hospital who were exposed to a COVID-19 patient. Information on demographic factors, medical history, exposure related factors and subsequently COVID-19 lab reports were collected. An exposure risk assessment designed collating various exposure related factors categorized the participants into those with high and low risk. We used logistic regression to estimate the odds ratio of our primary outcome, a positive COVID-19 test when the independent variables were exposure risk, age, gender and occupation.

Results: Among1858 frontline workers who were primary contacts of a COVID-19 patient at the hospital, 106 (5.7\%) incident reports of a positive COVID-19 test were recorded. None of the exposure related factors had any significant association with a positive COVID-19 test. However, high exposure risk category was significantly associated with COVID-19 positive test at the end of quarantine.

Conclusion: COVID-19 was more frequent among front-line health-care workers who belonged to high exposure category. Education at different levels of service delivery at hospitals is required for best practice in order to prevent COVID-19 among health care providers. There is need to develop additional strategies to ensure that the information is translated in to practice.
\end{abstract}

\section{Introduction}

The novel corona virus disease 2019 (COVID-19) is a global pandemic. Corona virus infections are emerging respiratory viruses that are known to cause illness ranging from the common cold to severe acute respiratory syndrome. It is a zoonotic pathogen that can be transmitted via animal-to-human and human-to-human interactions. About eight years after the MERS-CoV epidemic, the current outbreak of COVID-19 was first reported by the World Health Organization (WHO) on the 31st December 2019 and announced as a global pandemic on 11th March $2020 .^{1}$ The COVID-19 outbreak rapidly spread throughout the world causing a public health crisis globally. Healthcare workers (HCWs) are on the frontline of treating patients infected with COVID-19. People most at risk of acquiring the disease are those who are in contact with or care for patients with COVID-19. ${ }^{2}$ Personal exposure for closer and longer duration put the HCWs at higher risk of infection, increasing the probability for spread. ${ }^{3}$ Evidence of higher infection rates among health care workers during the severe acute respiratory syndrome (SARS) outbreak in 2002 stresses the need for protection of health care workers. ${ }^{4}$ Preliminary reports suggest that health-care workers account for $10-20 \%$ of all diagnoses. ${ }^{5}$ However, data related to its infection rate among HCWs are limited from low and middle income countries.

The health-work force is a valuable and scarce resource. Large number of COVID-19 affected health personnel getting isolated for treatment and their close contacts undergoing quarantine affects the hospital service delivery. ${ }^{6}$ It is important to understand the prevalence and risk factors for COVID-19 infection among HCWs to prevent depleting the workforce leading to critical shortages and negative

\footnotetext{
* Corresponding author.

E-mail address: cebp@pushpagiri.in (E. Mathew).
} 
impact on patient care, and in addition, the potential to transmit infection to vulnerable patients. ${ }^{7}$ Understanding the exposure related factors and risk associated with developing COVID-19 infection is critical for formulating and informing infection prevention and control (IPC) recommendations. ${ }^{2}$ Major factors for infection among HCWs include lack of understanding of the disease, inadequate use and availability of Personal Protective Equipment (PPE), uncertain diagnostic criteria, unavailability of diagnostic tests and psychological stress. Therefore, authorities should prioritize the protection of HCWs through education and training, improving the readiness of staff, incentives, availability of PPEs, and psychological support. ${ }^{8}$ Although HCWs are aware of infection control measures, low level of compliance with standard precautions in this group is frequent. Addressing the needs of health-care workers in the forefront of COVID-19 management is a high priority, however, evidence on using tools to identify those at high risk and association with infection at hospitals is scarce from developing countries. Hence, we conducted a study among frontline HCWs to determine the association between risk categorization of exposure to COVID -19 at work and getting infected with COVID-19.

\section{Materials and methods}

\subsection{Study design and participants}

We conducted a retrospective cohort study among the HCWs (18 years of age and above) who worked at the forefront to fight against COVID-19 since its outbreak in a single private tertiary care centre setting and having exposure with a confirmed COVID-19 patient. The participants in the cohort were doctors, nurses, technicians and other housekeeping staff from a tertiary care hospital in South Kerala, exposed to at least one COVID-19 positive patient while at work between July 2020 to January 2021. The designated hospital is a 900-bed multispeciality teaching hospital catering to the management of COVID-19 patients in addition to routine services.

\subsection{Data collection}

The participants were recruited from contact tracing database of the hospital during January-February 2021. A questionnaire was used to extract information from the database on the primary contacts of COVID-19 positive cases and it covered questions on socio-demographic characteristics, exposure related factors and lab reports (RT PCR) on COVID-19 infection. The risk assessment was done using the Ministry of Health guidelines for health care workers as follows. "High risk exposure: HCW or other person providing care to a COVID-19 case or labworker handling respiratory specimens from COVID-19 cases without recommended PPE or with possible breach of PPE; Performed aerosol generating procedures without appropriate PPE; HCWs without mask/ face-shield/goggles or having face to face contact with COVID-19 case within $1 \mathrm{~m}$ for more than $15 \mathrm{~min}$ or having accidental exposure to body fluids. Low risk exposure: Contacts who do not meet criteria of high risk exposure." ${ }^{9}$ Data were collected from the contact tracing database and online survey forms were used to collect the required information from the primary contacts due to the infectious nature of disease. Information from HCWs who could not be reached via online survey was collected using printed forms or telephonic interviews.

\subsection{Data analysis}

Categorical variables were described as frequencies and percentages and continuous variables presented as mean \pm standard deviation (SD) or median where appropriate. Chi-square was used to find the association between exposure related factors and COVID19 infection. Binary logistic regression was done with COVID-19 infection as the dependent variable and risk categories, age, gender and designation as independent variables. Analysis was done using SPSS (SPSS 25.0; SPSS Inc, Chicago,
IL, USA, IBM). A p-value $<0.05$ was considered statistically significant.

\subsection{Ethical considerations}

Approval from Institutional Ethics Committee was obtained. Confidentiality of participant identities was maintained throughout the study by keeping their information confidential.

\section{Results}

Among 1858 primary contacts to COVID-19 patient at work who were traced and quarantined, $106(5.7 \%)$ tested positive for the disease following the quarantine. Majority of the participants were women (86.3\%), and the mean age was $32.39 \pm 7.27$ years. The participants were doctors $(14.5 \%)$, nurses $(69.9 \%)$, technicians (3.6\%) and other housekeeping staff (12.1\%). The risk assessment of exposure identified $87.7 \%$ to be in low risk and $12.3 \%$ in high risk group. In both exposure groups, majority belonged to less than 40 years. (Table 1) Bivariate analysis did not show any significant association between sociodemographic and exposure related factors, and COVID-19 infection. Designation $(p=0.17)$, contact time $(p=0.53)$, distance from the source $(\mathrm{p}=0.10)$, gender $(\mathrm{p}=0.65)$, and hand hygiene $(\mathrm{p}=0.43)$ did not show any significant association with COVID -19 infection. (Table 2) Prevalence of infection was highest among those staying in the same hostel (9.4\%) followed by duty in radiology (8.3\%), labour room $(8.2 \%)$, COVID ward, intensive care unit (6.9\%) and medical intensive care unit (6.7\%). (Fig. 1) The exposure risk (categorized as high and low risk) assessed by collating various exposure related factors was found to be significantly associated with COVID -19 infection. While $14.9 \%$ of the primary contacts belonging to high risk group, turned positive, only $4.4 \%$ in the low risk group turned positive. In the model, the odds for COVID-19 infection was greater among those who had higher exposure when compared to those with lower exposure $(\mathrm{OR}=4.53$, p value $<$ $0.001,95 \% \mathrm{CI}=2.89-7.16$ ) after controlling for age, gender and designation (Table 3 ).

\section{Discussion}

In a retrospective cohort study among 1858 front line health-care workers in a tertiary care centre who were primary contacts to a COVID-19 case, we noted that COVID-19 infection was several fold higher in healthcare workers who belonged to high risk exposure group compared to low risk irrespective of age, gender or their designation at work. Protection of HCWs from infection is critical for the resilience of the health system facing a major pandemic like COVID-19.

Recent studies from the Netherlands and the UK reported prevalence rates in HCWs (all health care workers in the hospital) of $9 \%$ and $18 \%$, respectively ${ }^{10,11}$ compared to only $5.7 \%$ among primary contacts in our study. In this study, $87.7 \%$ of infected HCWs were females which is closer to that reported in the USA $(73 \%),{ }^{12}$ and by Glonar et al. ${ }^{13}$ in Iran

Table 1

Basic characteristics of the participants with respect to exposure risk.

\begin{tabular}{lllll}
\hline Sociodemographic factors & Low risk & High risk & Total & p value $^{\mathrm{a}}$ \\
\hline Age in years & & & & \\
$\quad 18-40$ & $1441(88.4)$ & $196(86.0)$ & $1637(88.1)$ & 0.003 \\
$\quad 10-60$ & $183(11.2)$ & $27(11.8)$ & $210(11.3)$ & \\
$\quad \begin{array}{l}\mathbf{2} 00 \\
\text { Gender }\end{array}$ & $6(0.4)$ & $5(2.2)$ & $11(0.6)$ & \\
Male & & & & \\
Female & $216(13.3)$ & $39(17.1)$ & $255(13.7)$ & 0.113 \\
Occupation & $1414(86.7)$ & $189(82.9)$ & $1603(86.3)$ & \\
Doctors & & & & \\
Nurses & $190(11.7)$ & $79(34.6)$ & $269(14.5)$ & \\
Technical & $1172(71.9)$ & $126(55.6)$ & $1298(69.9)$ & $<0.001$ \\
Others & $62(3.8)$ & $5(2.2)$ & $67(3.6)$ & \\
\hline
\end{tabular}

a Chi-square test. 
Table 2

Association between sociodemographic and exposure related factors, and COVID-19 test being positive among the primary contacts.

\begin{tabular}{|c|c|c|c|c|}
\hline \multirow[t]{2}{*}{ Variable } & \multicolumn{2}{|l|}{ COVID -19 } & \multirow[t]{2}{*}{ Total } & \multirow[t]{2}{*}{$\mathrm{p}$ value $\mathrm{a}^{\mathrm{a}}$} \\
\hline & Positive & Negative & & \\
\hline Low risk & $72(4.4)$ & $1558(95.6)$ & $1630(87.7)$ & $\mathrm{p}<0.001$ \\
\hline High risk & $34(14.9)$ & $194(85.1)$ & $228(12.3)$ & \\
\hline \multicolumn{5}{|l|}{ Gender } \\
\hline Male & $13(5.1)$ & $242(94.9)$ & $255(13.7)$ & 0.65 \\
\hline Female & $93(5.8)$ & $1510(94.2)$ & $1603(86.3)$ & \\
\hline \multicolumn{5}{|l|}{ Designation } \\
\hline Doctors & $11(4.1)$ & 258 (95.9) & 269 (14.5) & 0.17 \\
\hline Nurses & $72(5.5)$ & $1226(94.5)$ & $1298(69.9)$ & \\
\hline Technical & $7(10.4)$ & $60(89.6)$ & $67(3.6)$ & \\
\hline Others & $16(7.1)$ & 208 (92.9) & $224(12.1)$ & \\
\hline \multicolumn{5}{|c|}{ Source wearing mask } \\
\hline Yes & $14(3.9)$ & $345(96.1)$ & 359 (19.3) & 0.10 \\
\hline No & $92(6.1)$ & 1407 (93.9) & 1499 (80.7) & \\
\hline \multicolumn{5}{|c|}{ Distance from patient } \\
\hline$<1 \mathrm{~m}$ & $63(5.4)$ & $1094(94.6)$ & $1157(62.3)$ & 0.53 \\
\hline$>1 \mathrm{~m}$ & $43(6.1)$ & $658(93.9)$ & $701(37.7)$ & \\
\hline \multicolumn{5}{|c|}{ Duration of contact } \\
\hline$<15 \min$ & $38(5.3)$ & 679 (94.7) & $717(38.6)$ & 0.55 \\
\hline$>15 \mathrm{~min}$ & $68(6.0)$ & $1073(94.0)$ & $1141(61.4)$ & \\
\hline \multicolumn{5}{|l|}{ Hand hygiene } \\
\hline No & $12(7.0)$ & $159(93.0)$ & $171(9.2)$ & 0.43 \\
\hline Yes & $94(5.6)$ & $1593(94.4)$ & $1687(90.8)$ & \\
\hline \multicolumn{5}{|c|}{ Aerosol generating procedures } \\
\hline No & $87(5.4)$ & $1511(94.6)$ & $1598(86.0)$ & 0.23 \\
\hline Yes & $19(7.3)$ & $241(92.7)$ & $260(14.0)$ & \\
\hline \multicolumn{5}{|c|}{ Care to a COVID-19: breech of PPE } \\
\hline No & $101(5.7)$ & $1673(94.3)$ & $1774(95.5)$ & 0.92 \\
\hline Yes & $5(6.0)$ & $79(94.0)$ & $84(4.5)$ & \\
\hline \multicolumn{5}{|c|}{ Accidental exposure to fluids } \\
\hline Yes & $2(5.2)$ & $36(94.7)$ & $38(2.04)$ & 0.22 \\
\hline No & $88(5.4)$ & $1538(94.6)$ & $1626(87.5)$ & \\
\hline Do not know & $16(8.2)$ & $178(91.8)$ & $194(10.4)$ & \\
\hline
\end{tabular}

${ }^{\text {a }}$ Chi-square test.

(53.5\%). However, in contrast to our study in health workers, the proportion of women infected were much lower in a study on COVID-19 among general population in Oman. ${ }^{14}$ Majority of the participants infected were nurses which was concurrent with earlier study in Iran. ${ }^{13}$ Although each exposure related factor did not have any association with infection, combining and categorising them as high and low risk was found to be useful in identifying those likely to be infected. It may be assumed that it is not a single factor that acts as a risk for infection, but combination of risk behaviours and environments that leads to infection. Infection was higher in high risk group based on contact type at a University hospital in Italy. ${ }^{15} \mathrm{~A}$ study from China reported that high risk work areas, long duty hours, breech in hand hygiene were associated with COVID-19 infection. ${ }^{2}$ Healthcare workers are at increased risk of being exposed to viruses within hospitals and can also be a source of transmission to patients. Early identification of those at risk is important for further curbing the transmission. ${ }^{15}$ The prevalence of infection among primary contacts was highest among those staying in the same hostel probably due to lack of adherence to quarantine protocols. It is noteworthy that the prevalence was high among staff in radiology and labor room which emphasizes the risk of closed spaces in transmission and the need for appropriate protective measures. Gómez-Ochoa reported that the lack of information regarding exposure and risk of infection in different clinical settings limits the planning of appropriate preventive measures. ${ }^{16}$ Another study reported that health workers in patient settings and nursing homes had the greatest risk mostly due to inadequate PPE and reuse of PPE. ${ }^{17}$ Based on experience that viruses spread by respiratory droplets, the use of recommended personal protective equipment (PPE) is critical to reducing nosocomial transmission. ${ }^{18}$ Center for Disease Control and Prevention, United States (U. S.) recommends respirator use when caring for patients with suspected or confirmed COVID-19 patients and the universal use of masks at work place. $^{19}$

Our findings underscore the strict use of PPE and infection control measures regardless of workplace environment and whether patients or co-workers are known to be COVID-19 infected or not. This is critical to reduce infection rates among HCWs and to ensure reliable availability of the workforce in the current COVID-19 pandemic. Several factors suggested to be risk factors for infection among frontline HCWs are poor

Table 3

Association between risk category based on exposure and COVID-19 test being positive among primary contacts (odds ratios, confidence intervals and $\mathrm{p}$ values from binary logistic regression model).

\begin{tabular}{llll}
\hline Variables & Adjusted odds ratio & $95 \%$ CI & p value \\
\hline Exposure risk & & & \\
$\quad$ low risk & (Ref) & & \\
$\quad$ high risk & 4.53 & $2.89-7.16$ & $<0.001$ \\
Age & 0.98 & $0.96-1.02$ & 0.41 \\
Gender & & & \\
male & (Ref) & & \\
female & 1.14 & $0.58-2.23$ & 0.70 \\
Designation & & & \\
doctors & $($ Ref) & $1.02-4.23$ & 0.04 \\
nurses & 2.08 & $1.59-12.65$ & $<0.01$ \\
technicians & 4.50 & $1.29-7.16$ & 0.01 \\
$\quad$ housekeeping & 3.04 & & \\
\hline
\end{tabular}

Binary logistic regression model adjusted for age, gender and designation.

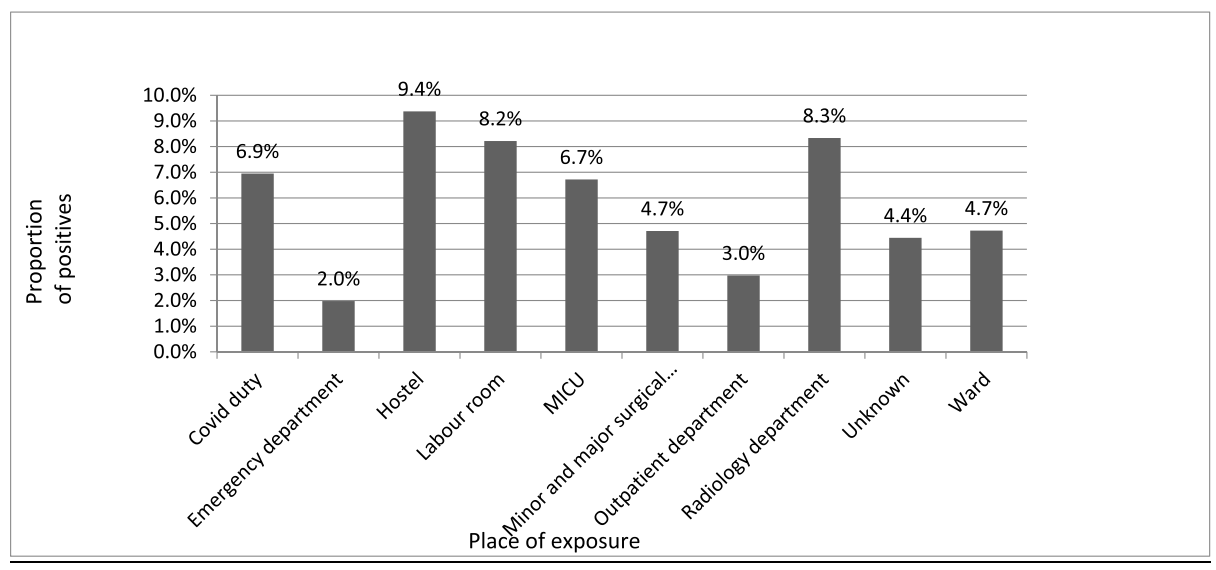

MICU - Medical intensive care unit

Fig. 1. Frequency of COVID-19 positives among primary contacts of cases with respect to the site of exposure MICU - Medical intensive care unit. 
understanding of the disease, lack of availability of Personal Protective Equipment (PPE), inadequate hand hygiene and use of PPE. ${ }^{20}$ It is recommended that, the protection of HCWs by authorities should be prioritized providing health education, training in knowledge and skills including the type of PPEs, their proper use, cleaning, re-use and disposal. In addition, stable and adequate PPE supply is highly recommended. ${ }^{21}$

To our knowledge, this is the first study from India to understand the role of exposure risk categorization and COVID infection among primary occupational contacts of COVID-19 patients. It was possible as regular contact tracing was carried out efficiently within the hospital. Using both online platform and personal interviews improved the response rate and identification of majority of primary contacts. However, there are limitations for the study. First, there may be an underestimation of cases. The contacts might have developed symptoms and turned positive after the quarantine period which may not be reflected in the study. Secondly, the possibility that infections were acquired from the community rather than from within the hospital which could not be differentiated. In at least some cases, family members of the HCWs were affected by COVID-19 over the same period. Moreover, the results are from a single centre, hence cannot be generalized.

\section{Conclusion}

Front-line health-care workers with high exposure were at greater risk for COVID-19 infection. There is need for designing preventive measures appropriate for different clinical settings at the hospital and education of personnel at all levels in health delivery. Further research is required to understand the evolving implications of this pandemic among front-line health-care workers.

\section{Funding}

This research did not receive any specific grant from funding agencies in the public, commercial, or not-for-profit sectors.

\section{Declaration of competing interest}

The authors declare that they have no competing interests.

\section{Acknowledgements}

We express our deep sense of gratitude to the Department of Community Medicine, Pushpagiri Institute of Medical Sciences, Tiruvalla, Kerala for all the support.

\section{Appendix A. Supplementary data}

Supplementary data to this article can be found online at https://doi. org/10.1016/j.cegh.2021.100933.

\section{References}

1 World Health Organization. Risk Assessment and Management of Exposure of Health Care Workers in the Context of COVID-19: Interim Guidance, 19 March 2020 [Internet]. World Health Organization; 2020 [cited 2021 Nov 3]. Report No.: WHO/2019-nCov/ HCW_risk_assessment/2020.2. Available from https://apps.who.int/iris/handle/ 10665/331496. Accessed November 3, 2021.

2 Ran L, Chen X, Wang Y, Wu W, Zhang L, Tan X. Risk factors of healthcare workers with coronavirus disease 2019: a retrospective cohort study in a designated hospital of wuhan in China. Clin Infect Dis. 2020;71(16):2218-2221.

3 Black JRM, Bailey C, Przewrocka J, Dijkstra KK, Swanton C. COVID-19: the case for health-care worker screening to prevent hospital transmission. Lancet. 2020;395 (10234):1418-1420.

4 Wu Z, McGoogan JM. Characteristics of and important lessons from the coronavirus disease 2019 (COVID-19) outbreak in China: summary of a report of 72314 cases from the Chinese center for disease control and prevention. J Am Med Assoc. 2020; 323(13):1239-1242. Apr 7.

5 Lazzerini M, Putoto G. COVID-19 in Italy: momentous decisions and many uncertainties. The Lancet Global Health. 2020;8(5):e641-e642.

6 Lu W, Danni Y, Xinlan W, Yujuan C, You L, Huai Y. Correlation between hand hygiene compliance and nosocomial infection in medical staff. Chin J Disinfect. 2014; 31:1237-1238.

7 Kulkarni A, Khasne RW, Dhakulkar BS, Mahajan HC. Burnout among healthcare workers during COVID-19 pandemic in India: results of a questionnaire-based survey. Indian J Crit Care Med. 2020;24(8):664-671.

8 Ali S, Noreen S, Farooq I, Bugshan A, Vohra F. Risk assessment of healthcare workers at the frontline against COVID-19. Pakistan J Med Sci. 2020;36:S99-S103.

9 Advisory for Managing Health Care Workers Working in COVID and Non-COVID Areas of the Hospital Ministry of Health \& Family Welfare Directorate General of Health Services (EMRDivision); 2020. Available from https://www.mohfw.gov.in/pdf/Advisoryform anagingHealthcareworkersworkinginCOVIDandNonCOVIDareasofthehospital.pdf.

10 Tostmann A, Bradley J, Bousema T, et al. Strong associations and moderate predictive value of early symptoms for SARS-CoV-2 test positivity among healthcare workers, The Netherlands, March 2020. Euro Surveill. 2020;25(16):2000508.

11 Keeley AJ, Evans C, Colton H, et al. Roll-out of SARS-CoV-2 testing for healthcare workers at a large NHS Foundation Trust in the United Kingdom, March 2020. Euro Surveill. 2020;25(14):2000433. https://doi.org/10.2807/1560-7917. ES.2020.25.14.2000433.

12 CDC COVID-19 Response Team. Characteristics of health care personnel with COVID19-United States, February 12-April 9, 2020. MMWR (Morb Mortal Wkly Rep). 2020;69. April(15)). Available from: https://www.cdc.gov/mmwr/volumes /69/wr/mm6915e6.htm.

13 Sabetian G, Moghadami M, Hashemizadeh Fard Haghighi L, et al. COVID-19 infection among healthcare workers: a cross-sectional study in southwest Iran. Virol J. 2021;18(1):58. Mar 17.

14 Khamis F, Al Rashidi B, Al-Zakwani I, Al Wahaibi AH, Al Awaidy ST. Epidemiology of COVID-19 infection in Oman: analysis of the first 1304 cases. Jun 30 Oman Med J. 2020;35(3):e145. https://doi.org/10.5001/omj.2020.60. Available from.

15 Vimercati L, De Maria L, Quarato M, et al. COVID-19 hospital outbreaks: protecting healthcare workers to protect frail patients. An Italian observational cohort study. Int J Infect Dis. 2021;102:532-537.

16 Gómez-Ochoa SA, Franco OH, Rojas LZ, et al. COVID-19 in health-care workers: a living systematic review and meta-analysis of prevalence, risk factors, clinical characteristics, and outcomes. Am J Epidemiol. 2021;190(1):161-175.

17 Nguyen LH, Drew DA, Graham MS, et al. Risk of COVID-19 among front-line healthcare workers and the general community: a prospective cohort study. Sep. 1 Lancet Public Health. 2020;5(9):e475-e483.

18 Verbeek JH, Rajamaki B, Ijaz S, et al. Personal protective equipment for preventing highly infectious diseases due to exposure to contaminated body fluids in healthcare staff. Cochrane Database Syst Rev. 2020;4(4):CD011621.

19 Center for Disease Control and Prevention. Interim Infection Prevention and Control Recommendations for Patients with Suspected of Confirmed Coronavirus Disease 2019 (COVID-19) in Health Care Settings; 2020. Available from https://www.cdc.gov/cor onavirus/2019-ncov/hcp/infection-control-recommendations.html. Accessed April $13,2020$.

20 Mehta S, Machado F, Kwizera A, et al. COVID-19: a heavy toll on health-care workers. Lancet Resp Med. 2021;9(3):226-228.

21 Al Thobaity A, Alshammari F. Nurses on the frontline against the COVID-19 pandemic: an integrative review. DMJ. 2020;3(3):87-92. 